\title{
Could Diabetic Foot Be Predicted by Plantar Temperature Using Infrared Thermography?
}

\author{
Dong Soo Kim ${ }^{1}$, Shin Kyoung $\mathrm{Kim}^{2}$ and Manyong $\mathrm{Choi}^{3}$, Jong Moon Kim ${ }^{2}$
}

${ }^{1}$ Biochemistry, Baylor University, Waco, TX, USA

${ }^{2}$ Physical Medicine and Rehabilitation, Konkuk University, Chungju, Republic of Korea

${ }^{3}$ Korea Research Institute of Standards and Science, Daejun, Republic of Korea

rmjmk@kku.ac.kr

Background and aims: To evaluate the foot plantar temperature in normal and diabetic patient (DM) and to assess development of diabetic foot ulceration could be predicted by the foot temperature measurement with infrared thermography (IR).

Materials and methods: The first investigation was designed for assessment of correlation between foot plantar temperature and diabetic foot. Eighteen normal controls were compared to $23 \mathrm{DM}$ without polyneuropathy (Group I), 20 DM with polyneuropathy (Group II), and 16 DM with polyneuropathy plus diabetic foot ulcer (Group III). Temperature were assessed using IR in six standard plantar sites; medial longitudinal arch (MLA), lateral longitudinal arch (LLA), heel, 5th toe, 3rd metatarsal head (3MTH), and greater toe (GT), and mean foot temperature; MFT. The second investigation would be followed after 5 years among the same subjects except for control group.

Results: Sixteen Group I subjects were contacted but ten accepted the follow-up investigation. Thirteen of fifteen contacted Group II and eight of eleven contacted Group III subjects accepted to be enrolled in the 2 nd investigation. In the 1 st investigation, the duration of diabetes was significantly longer in Group II and Group III than in Group I $(p<0.05)$. The MFT was significantly higher in Group III (33.18 $\left.\pm 1.86^{*}\right)$ than in Group II (30.89 $\left.\pm 1.51^{*}\right)$ and higher in Group II than in Group I $(29.13 \pm 1.63 *)(\mathrm{p}<0.001)$, but no significance was shown between Group I and the control group. There was an increasing tendency of focal MFT at $3 \mathrm{MTH}$, MLA and GT in DM. In the 2nd investigation, six of 10 previous Group I developed polyneuropathy (into Group II), and three of 13 previous Group II and 1 of 10 previous Group I had foot ulcer (into Group III). Temperature of 3MTH in Group II (29.87 $\pm 1.21^{*}$ ) and Group III (32.51 $\left.\pm 1.41^{*}\right)$ had showed significantly higher than that of Group I (29.03 \pm 1.26$)$ and Group II (31.29 \pm 1.33$)$ in the 1st investigation $(\mathrm{p}<0.05)$.

Conclusion: Regional temperature of 3MTH, GT and MLA were higher in DM according to microangiopathy followed by arteriovenous shunt, and these, especially which of 3MTH could be the predictable value of developing diabetic foot.

Keywords: diabetic foot, thermography, plantar temperature, polyneuropathy 